\title{
Sex-specific impact of asthma during pregnancy on infant gut microbiota
}

\author{
Petya T. Koleva ${ }^{1}$, Hein M. Tun ${ }^{1}$, Theodore Konya ${ }^{2}$, David S. Guttman ${ }^{3}$, \\ Allan B. Becker ${ }^{4}$, Piush J. Mandhane', Stuart E. Turvey ${ }^{5}$, Padmaja Subbarao ${ }^{6}$, \\ Malcolm R. Sears ${ }^{7}$, James A. Scott ${ }^{2}$ and Anita L. Kozyrskyj ${ }^{1}$, for the CHILD \\ Study Investigators ${ }^{8}$
}

Affiliations: ${ }^{1}$ Dept of Pediatrics, University of Alberta, Edmonton, AB, Canada. ${ }^{2}$ Dalla Lana School of Public Health, University of Toronto, Toronto, ON, Canada. ${ }^{3} \mathrm{Cell}$ and Systems Biology, University of Toronto, Toronto, ON, Canada. ${ }^{4}$ Dept of Pediatrics and Child Health, University of Manitoba, Winnipeg, MB, Canada. ${ }^{5}$ Dept of Pediatrics, University of British Columbia, Vancouver, BC, Canada. ${ }^{6}$ Dept of Pediatric Respiratory Medicine, University of Toronto, Toronto, ON, Canada. ${ }^{7}$ Dept of Medicine, McMaster University, Hamilton, ON, Canada. ${ }^{8} \mathrm{~A}$ full list of the CHILD (Canadian Healthy Infant Longitudinal Development Study) Study Investigators and their affiliations can be found in the Acknowledgements section.

Correspondence: Anita Kozyrskyj, 3-527 Edmonton Clinic Health Academy, 11405-87 Avenue, Edmonton, AB, Canada. E-mail: kozyrskyqualberta.ca

@ERSpublications

Gut lactobacilli are less abundant at 3-4 months in male but not female infants following maternal prenatal asthma http://ow.ly/jXnl30fU9xH

Cite this article as: Koleva PT, Tun HM, Konya T, et al. Sex-specific impact of asthma during pregnancy on infant gut microbiota. Eur Respir J 2017; 50: 1700280 [https://doi.org/10.1183/13993003.00280-2017].

ABSTRACT Asthma during pregnancy is associated with retardation of fetal growth in a sex-specific manner. Lactobacilli microbes influence infant growth. This study aimed to determine whether lactobacilli and other microbes are reduced in the gut of infants born to an asthmatic mother, and whether this differs by the sex of the infant.

Mother-infant pairs ( $\mathrm{N}=1021)$ from the Canadian Healthy Infant Longitudinal Development full-term cohort were studied. The abundance of infant faecal microbiota at 3-4 months, profiled by gene sequencing, was compared between both women with and without asthma treatment during pregnancy. Infant sex, maternal ethnicity, pre-pregnancy overweight and atopy status, birth mode, breastfeeding status and intrapartum antibiotic treatment were tested as covariates.

Independent of birth mode and other covariates, male, Caucasian infants born to women with prenatal asthma harboured fewer lactobacilli in the gut at 3-4 months of age. If asthmatic mothers had prepregnancy overweight, the abundance of Lactobacillus in males was further reduced in the infant gut, whereas the microbiota of female infants was enriched with Bacteroidaceae. Similar differences in infant gut microbial composition according to maternal prenatal asthma status were also more evident among women with food or environmental allergies.

Gut lactobacilli were less abundant in male infants, but Bacteroidaceae were more abundant in female infants at 3-4 months of age, following maternal asthma during pregnancy.

This article has supplementary material available from erj.ersjournals.com

Received: Feb 082017 | Accepted after revision: Aug 162017

Support statement: The Canadian Institutes of Health Research (CIHR) and the Allergy, Genes and Environment (AllerGen) Network of Centres of Excellence provided core support for the CHILD study. The study was specifically funded by the CIHR Microbiome Initiative team grant, \#227312. Funding information for this article has been deposited with the Crossref Funder Registry

Conflict of interest: None declared.

Copyright $\odot$ ERS 2017. This ERJ Open article is open access and distributed under the terms of the Creative Commons Attribution Non-Commercial Licence 4.0. 


\section{Introduction}

Asthma is a common chronic disorder during pregnancy, affecting between $8 \%$ and $13 \%$ of pregnant women [1]; it is often a comorbid condition with food and environmental allergies [2]. Asthma exacerbation rates during pregnancy are high [3], and more severe asthma or poor symptom control have been associated with low birthweight [4-7]. A greater risk for childhood asthma following uncontrolled versus controlled maternal asthma during pregnancy has been reported [8]. These variations in risk to the offspring point to a potential in utero influence of maternal asthma. Asthma during pregnancy often leads to maternal antibiotic treatment and delivery by caesarean $[7,9]$, interventions that can interrupt the transfer of maternal lactobacilli, which become more prominent in the placenta, gut and vaginal tract in the last trimester of pregnancy [10-13]. Reduced abundance of Lactobacillales has been observed in the meconium of newborns of mothers with atopic disease [14].

Female infants are generally born smaller than male infants; however, in response to maternal asthma, the Caucasian male fetus is more likely to have a lower birthweight and show growth retardation when delivered at term than the female fetus [5]. MarTin et al. [15] recently reported less colonisation of stool with Lactobacillus species at birth and during the first month of life among full-term male infants in comparison to female infants. Postnatally, depending on the Lactobacillus species, since weight promotion is species-specific, the dominance of lactobacilli in the infant gut will determine the extent of weight gain and subsequent atopic disease [16-18]. Altogether, evidence is mounting for a programming role of prenatal asthma that might affect the infant gut microbiome. These observations led us to study the influence of maternal asthma status during pregnancy on infant intestinal microbiota in the Canadian Healthy Infant Longitudinal Development (CHILD) general population birth cohort. Specifically, we determined whether the genus Lactobacillus was reduced in the gut of infants born to an asthmatic mother, especially in the gut of male infants. Second, we sought evidence for a fetal programming role of maternal asthma that was independent of birth mode and related events, and was enhanced by maternal comorbid conditions, such as overweight and atopic disease.

\section{Material and methods}

\section{Study design and sample collection}

This study was conducted on 1021 mothers and their infants who participated in the Canadian Healthy Infant Longitudinal Development (CHILD) Study - a national population-based birth cohort (www. canadianchildstudy.ca) [19-21]. Pregnant women were recruited in their second or third trimester at the Manitoba, Edmonton (Alberta) and Vancouver (Ontario) sites of the CHILD cohort. Written informed consent was obtained from all mothers. This microbiome study of the CHILD cohort was approved by the Human Research Ethics Boards of the University of Alberta (\#Pro00010073). Hospital birth records provided data on mode of delivery, sex and infant exposures during birth. The mothers' anthropometry pre-pregnancy and infant diets were obtained from standardised questionnaires and hospital records. Asthma during pregnancy was defined as present, if mothers had responded 'yes' to the question 'Have you had any treatment for asthma or wheezing in the last 12 months?' in prenatal questionnaires. Any treatment for asthma, as indicated by hospitalisation, a physician's visit or medication use, has been validated as a measure for asthma morbidity during pregnancy [22]. The 12-month assessment period of asthma treatment included the time just prior to conception. Since $23-30 \%$ of asthmatic women discontinue treatment during pregnancy due to lessening symptoms, [22, 23] the CHILD study questionnaire would have correctly classified women reporting treatment prior to conception but not during pregnancy.

Data on maternal atopic status during pregnancy was also obtained from prenatal questionnaires as symptoms in response to one or more food or environmental allergens, such as hay fever, atopic dermatitis, and allergies to pollens, grasses, trees, weeds, food, insects or pets. Faecal samples were obtained from infants at 3-4 months of age for microbial profiling. Parents were instructed to refrigerate soiled diapers prior to retrieval by a CHILD research assistant during a home visit. Faecal samples were then transported to the laboratory in a cooler, where they were divided into aliquots and stored at $-80^{\circ} \mathrm{C}$ prior to DNA extraction and sequencing.

\section{Sequencing and data processing}

Pooled 16S rRNA fragments were sequenced using the MiSeq Illumina sequencing method at the Centre for the Analysis of Genome Evolution \& Function at the University of Toronto. Sequences were de-multiplexed and binned into individual samples according to their barcodes using the QIIME pipeline (version 1.8.0). The sequences were then quality filtered by removing reads having more than 10 sites with a Phred quality score less than 20. Using the closed-picking algorithm in QIIME, sequences that passed quality control were clustered into operational taxonomic units (OTUs) at a 97\% similarity cut-off. Finally, OTUs were aligned with the Greengenes bacterial reference database (v. 13.5) to identify microbes at the 
phylum, family and genus levels. The OTUs with an overall relative abundance below 0.0001 were excluded from subsequent analyses [24]. After cleaning and processing, the total number of unique OTUs was 1325 and the total number of reads was 270 million (median $2.3 \times 10^{5}$ per sample, range $6.7 \times 10^{3}$ $8.3 \times 10^{6}$ ). Data were rarefied to 13000 sequences per sample. The metrics of OTU (microbial) relative abundance and diversity (Shannon and Simpson diversity indices, Chaol species richness index) were generated in QIIME to profile infant gut microbiota in individual faecal samples.

\section{Statistical analysis}

The statistical analysis of data was performed with the Statistical Analysis Systems (SAS) Software (version 9.4.; SAS Institute Inc., Cary, NC, USA). Relative abundance of microbial taxa (at the phylum, family or genus level) and biodiversity measures (Chaol, Shannon and Simpson indices) were presented as medians and interquartile ranges, because these measures were not normally distributed. Medians were compared using the non-parametric Wilcoxon rank sum test or the Kruskal-Wallis test for $>2$ group comparisons, with statistical significance at $\mathrm{p}<0.05$. Although the hypothesis regarding lactobacilli was a priori, the Benjamini-Hochberg method to adjust $\mathrm{p}$-values for the false discovery rate (FDR, where $\mathrm{p}<\mathrm{FDR} \mathrm{p}$ ) was applied to analyses for all bacterial taxa with the SAS multi-test procedure [25]. As gut microbiome differences have been reported between European/North American and Asian populations [26], analyses were restricted to mothers of Caucasian ethnicity. Restriction to Caucasian ethnicity also allowed us to test associations within a population at highest risk for infant wheeze: male Caucasian infants [27].

To test the a priori research question on infant sex and adjust for putative confounding factors, analyses were stratified by infant sex, birth mode, breastfeeding status and intrapartum antibiotic exposure (also a strong marker for infant antibiotic use; [28]). Using the adjusted rank transform (ART) nonparametric test for interaction [29], the p-value for the interaction between infant sex and maternal prenatal asthma on Lactobacillus abundance was 0.001 . To determine the impact of maternal comorbid conditions, statistical testing was also performed following stratification by maternal prenatal atopy status and pre-pregnancy weight (normal weight $\leqslant 24.9$; overweight $\geqslant 25.0$ body mass index). Independence of association between maternal asthma during pregnancy, and highest or lowest tertile Lactobacillus levels was further determined using purposeful stepwise logistic regression modelling, adjusting for confounding factors. To identify discriminative taxonomic biomarkers, the linear discriminant analysis effect size (LEfSe) was conducted with a linear discriminant analysis (LDA) log score cut-off of 2, followed by the Kruskal-Wallis test with the Dunn's multiple comparison test, using no prenatal maternal asthma as the reference group.

\section{Results}

$9 \%$ of the infants under study were born to women with asthma during pregnancy, the majority by vaginal delivery; $8 \%$ of these infants were born by emergency caesarean and $7 \%$ by elective caesarean. Similar to the whole cohort, 79\% of infants born to asthmatic mothers were fed breast milk at 3-4 months. Prenatal maternal asthma was not differentially distributed by infant sex or ethnicity, birth mode, early term birth, birthweight, breastfeeding status, maternal pre-pregnancy weight and maternal intrapartum antibiotic treatment (table 1); it tended to be higher in both the lowest and highest infant weight gain tertiles. Food and environmental allergies during pregnancy were more common among women with prenatal asthma than among those without asthma. Compared to the complete CHILD birth cohort [20], our CHILD study population of 1021 mother-infant pairs was similar in terms of male sex (53\% versus 52\%), Caucasian ethnicity (78\% versus $73 \%$ ) and atopic status of the mother (61\% versus $58 \%)$.

Gut microbiota of all infants at 3-4 months of age were dominated by members of the phyla Bacteroidetes, Firmicutes and Proteobacteria (supplementary figure S1). Microbiota in female infants tended to be enhanced with Bacteroidetes (median relative abundance, 20.8\% versus 9.3\%). With the exception of Firmicutes being less abundant in female infants born to women with asthma $(p=0.03)$, no other differences were evident in phylum abundance, microbial richness or diversity between infants born to women with versus those without prenatal asthma (supplementary figure S1, supplementary table S1). Crude differences in median and high abundance were found for gut colonisation by members of the genus Lactobacillus, according to infant feeding and asthma status of the mother (table 1).

The abundance of Lactobacillus was significantly lower among infants born to women with non-Caucasian ethnicity and to women with asthma during pregnancy (table 1). Based on the ART test, the p-value for the interaction term between prenatal asthma and Caucasian ethnicity was 0.002 . In Caucasian infants, prenatal maternal asthma was significantly associated with a lower relative abundance of the genus Lactobacillus (median $0.001 \%$ versus $0.002 \%, \mathrm{p}=0.01$; table 2 ). This genus was not differentially abundant by maternal asthma status in non-Caucasian infants (data not shown). Stratification by infant sex yielded statistical significance for a reduction in gut Lactobacillus species among Caucasian male infants (median $0.002 \%$ versus $0.0005 \%, \mathrm{p}=0.02$ ), but not female infants born to women with asthma. No differences in gut 
TABLE 1 Distribution of maternal prenatal asthma status and infant faecal Lactobacillus abundance according to study covariates

\begin{tabular}{|c|c|c|c|c|c|c|c|}
\hline & \multirow[t]{2}{*}{ Prevalence } & \multicolumn{2}{|c|}{$\begin{array}{l}\text { Proportion with } \\
\text { maternal prenatal } \\
\text { asthma }{ }^{\#}\end{array}$} & \multicolumn{2}{|c|}{$\begin{array}{l}\text { Median abundance } \\
\text { (IQR) of Lactobacillus } \\
\text { species }\end{array}$} & \multicolumn{2}{|c|}{$\begin{array}{c}\text { Proportion with } \\
\text { highest Lactobacillus } \\
\text { abundance }\end{array}$} \\
\hline & & & p-value I $^{2}$ & & p-value ${ }^{+}$ & & p-value ? \\
\hline \multicolumn{7}{|c|}{ Mothers } & \\
\hline \multicolumn{8}{|c|}{ Maternal asthma during pregnancy } \\
\hline \multicolumn{8}{|l|}{ Site } \\
\hline Edmonton & 345 (34) & $34(10)$ & 0.47 & $0.0009(0.0-8.8)$ & 0.02 & $88(26)$ & 0.90 \\
\hline Winnipeg & $307(30)$ & $22(7)$ & & $0.002(0.0-17.6)$ & & $78(25)$ & \\
\hline Vancouver & $369(36)$ & $31(8)$ & & $0.001(0.0-4.5)$ & & $89(24)$ & \\
\hline \multicolumn{8}{|l|}{ Maternal ethnicity§ } \\
\hline Caucasian & 795 (78) & 74 (9) & 0.07 & $0.002(0.0-17.6)$ & 0.003 & $209(26)$ & 0.03 \\
\hline No & 375 (37) & $11(3)$ & & $0.002(0.0-6.7)$ & & $103(27)$ & \\
\hline \multicolumn{8}{|c|}{ Maternal pre-pregnancy overweight status $\S$} \\
\hline Normal weight & 587 (57) & $43(7)$ & 0.20 & $0.002(0.0-17.6)$ & 0.18 & $154(26)$ & 0.41 \\
\hline Overweight/Obese & 396 (39) & $39(10)$ & & $0.001(0.0-7.4)$ & & 94 (24) & \\
\hline \multicolumn{8}{|l|}{ Infants } \\
\hline \multicolumn{8}{|l|}{ Sex } \\
\hline Female & $476(47)$ & $46(10)$ & 0.26 & $0.001(0.0-10.4)$ & 0.93 & $113(24)$ & 0.43 \\
\hline Male & $545(53)$ & $41(8)$ & & $0.001(0.0-17.6)$ & & $142(26)$ & \\
\hline \multicolumn{8}{|c|}{ Antibiotic intrapartum exposure ${ }^{\S}$} \\
\hline Exposure & $434(44)$ & $42(10)$ & 0.36 & $0.001(0.0-17.6)$ & 0.39 & $106(24)$ & 0.94 \\
\hline No exposure & $560(56)$ & $44(8)$ & & $0.002(0.0-10.4)$ & & $138(25)$ & \\
\hline \multicolumn{8}{|l|}{ Mode of delivery $\S$} \\
\hline Vaginal & $760(74)$ & 70 (9) & 0.48 & $0.001(0.0-0.6)$ & 0.31 & $179(24)$ & 0.19 \\
\hline \multicolumn{8}{|c|}{ Breastfeeding status at 3 months ${ }^{\S}$} \\
\hline Breastfed & $828(81)$ & $69(8)$ & 0.56 & $0.003(0.0-17.6)$ & $<0.0001$ & $246(30)$ & $<0.0001$ \\
\hline Not breastfed & $187(18)$ & $18(10)$ & & $0.002(0.0-7.4)$ & & $6(3)$ & \\
\hline \multicolumn{8}{|l|}{ Weight gain (medium tertile) } \\
\hline Yes & 334 (34) & $21(6)$ & 0.06 & $0.002(0.0-0.05)$ & 0.09 & $88(26)$ & 0.44 \\
\hline No & $662(66)$ & $66(10)$ & & $0.001(0.0-0.03)$ & & $160(24)$ & \\
\hline
\end{tabular}

Data are presented as $\mathrm{n}(\%)$, unless otherwise stated. IQR: interquartile range. p-values $<0.05$ are indicated in bold. ${ }^{\#}$ : percentage of asthma and highest Lactobacillus abundance within category; ": comparison of proportions was performed using Fisher's exact test for $2 \times 2$ and $2 \times 3$ contingency tables; ${ }^{+}$: medians were compared with the Wilcoxon rank sum test or Kruskal-Wallis test for $>2$ group comparisons; ${ }^{\S}$ : variables with missing information for some infants.

Lactobacillus abundance were found between non-Caucasian males born to mothers with (median $0.0005 \%, \mathrm{n}=5$ ) and without (median $0.0007 \%, \mathrm{n}=112$ ) prenatal asthma.

To reduce bias from perinatal exposures, comparisons were conducted only within vaginally delivered, breastfed infants, who were not exposed to maternal antibiotics during birth. The restricted analyses revealed similar trends for the genus Lactobacillus in the gut microbiota of male, Caucasian infants ( $p=0.06$ ) born to an asthmatic mother (table 3, supplementary table S2). Other sex-specific differences were uncovered. Following prenatal asthma, the gut of female, Caucasian infants, born vaginally and breastfed, without exposure to maternal intrapartum antibiotics, was more abundant in members of the 
TABLE 2 Median relative abundance of dominant bacterial taxa at the phylum, family and genus levels in infant gut microbiota at 3-4 months, according to maternal prenatal asthma in all Caucasian infants

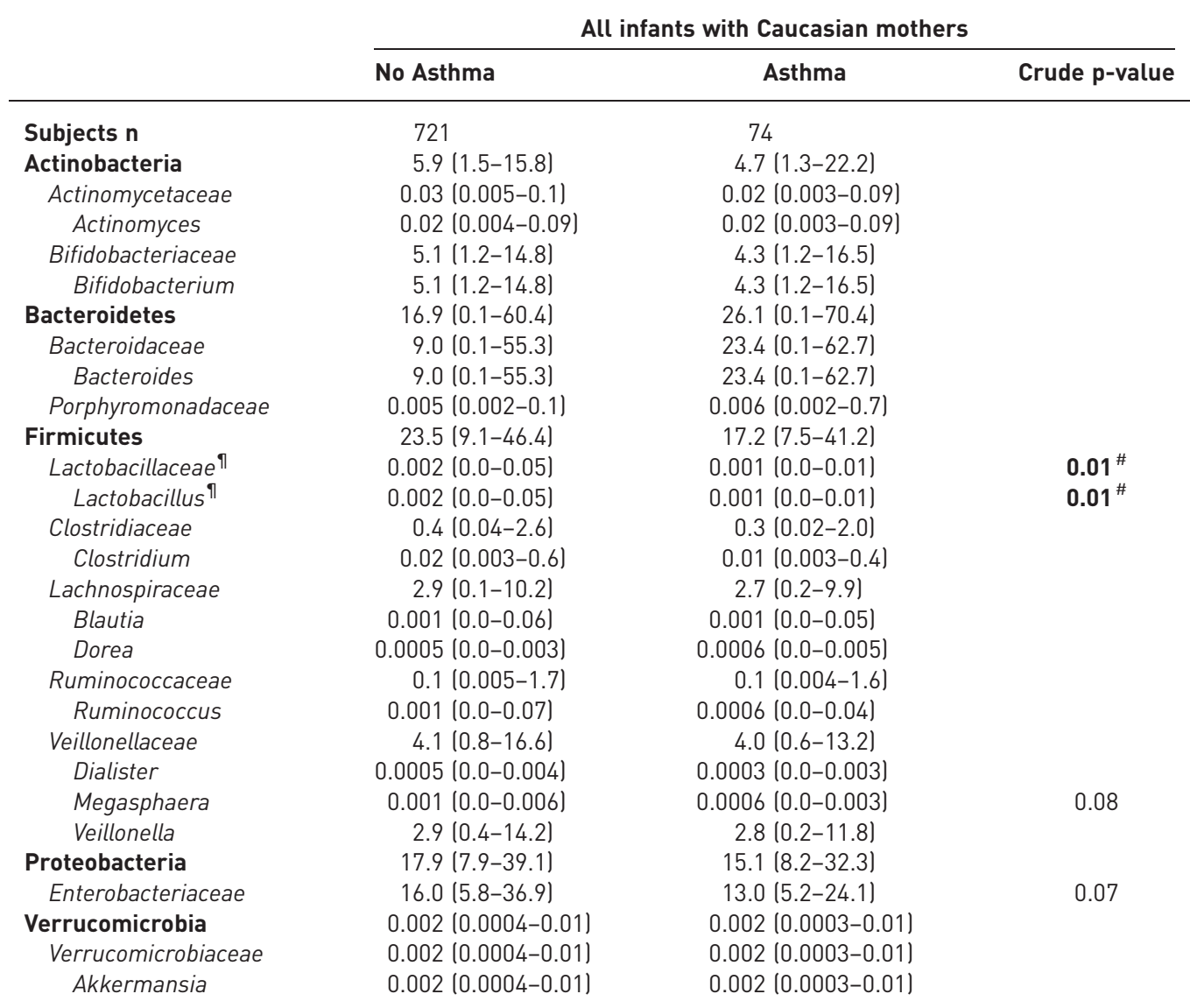

Data are presented as median (interquartile range), unless otherwise stated. Comparisons were performed using the Wilcoxon rank sum test. The false discovery rate (FDR) was used to adjust $p$-values for multiple testing and crude $p$-values <FDR p-values are indicated by "\#". The $p$-values $<0.1$ are reported and $p$-values $<0.05$ are indicated in bold. ๆ : comparison of Lactobacillus was the primary hypothesis.

Bacteroidetes phylum ( $\mathrm{p}=0.02$, table 3 ) and less abundant in Enterobacteriaceae $(\mathrm{p}=0.03)$. Differences were also evident at the Bacteroidaceae family level ( $\mathrm{p}=0.02$, supplementary table $\mathrm{S} 2$ ) in all female breastfed infants born vaginally without intrapartum antibiotics, alongside a reduction in abundance of the Firmicutes phylum $(\mathrm{p}<0.05)$.

Lactobacilli were less abundant in male infants born to women with asthma and concomitant food or environmental allergies, or asthma and pre-pregnancy overweight; statistical significance was not observed in the absence of either comorbidity (table 4). Based on the ART test, the p-value for the interaction term between prenatal asthma and infant sex on Lactobacillus abundance was 0.003 for maternal atopy comorbidity and 0.06 for overweight comorbidity. Following the same maternal asthma comorbidity during pregnancy, the abundance of Bacteroidaceae was substantially and significantly higher in female infants. The p-value for this sex interaction term was 0.001 for maternal atopy comorbidity and 0.002 for overweight (ART test). Firmicutes were much less abundant in females born to overweight mothers with asthma. Within the Firmicutes phylum, the genus Megasphaera was less abundant in the infant gut following maternal asthma comorbid with overweight or atopy, and this difference was statistically significant in females of mothers with atopic asthma (table 4). The p-value for the sex interaction term for differences in Megasphera abundance related to maternal atopic asthma was 0.14 (ART test). Following atopic asthma during pregnancy, Enterobacteriaceae were less common in the gut microbiota of offspring, more so in males than females; only Bacteroidaceae enrichment survived correction for multiple testing. With one exception (when the crude p-value was 0.06 with reduced sample size following restriction, as presented in table 3 ), reductions in Lactobacillus abundance were also statistically significant after the FDR correction. 
TABLE 3 Median relative abundance of dominant bacterial taxa at the phylum, family and genus levels in infant gut microbiota at 3-4 months, by maternal prenatal asthma and infant sex in Caucasian, vaginally delivered, breastfed infants, not exposed to maternal antibiotics during birth

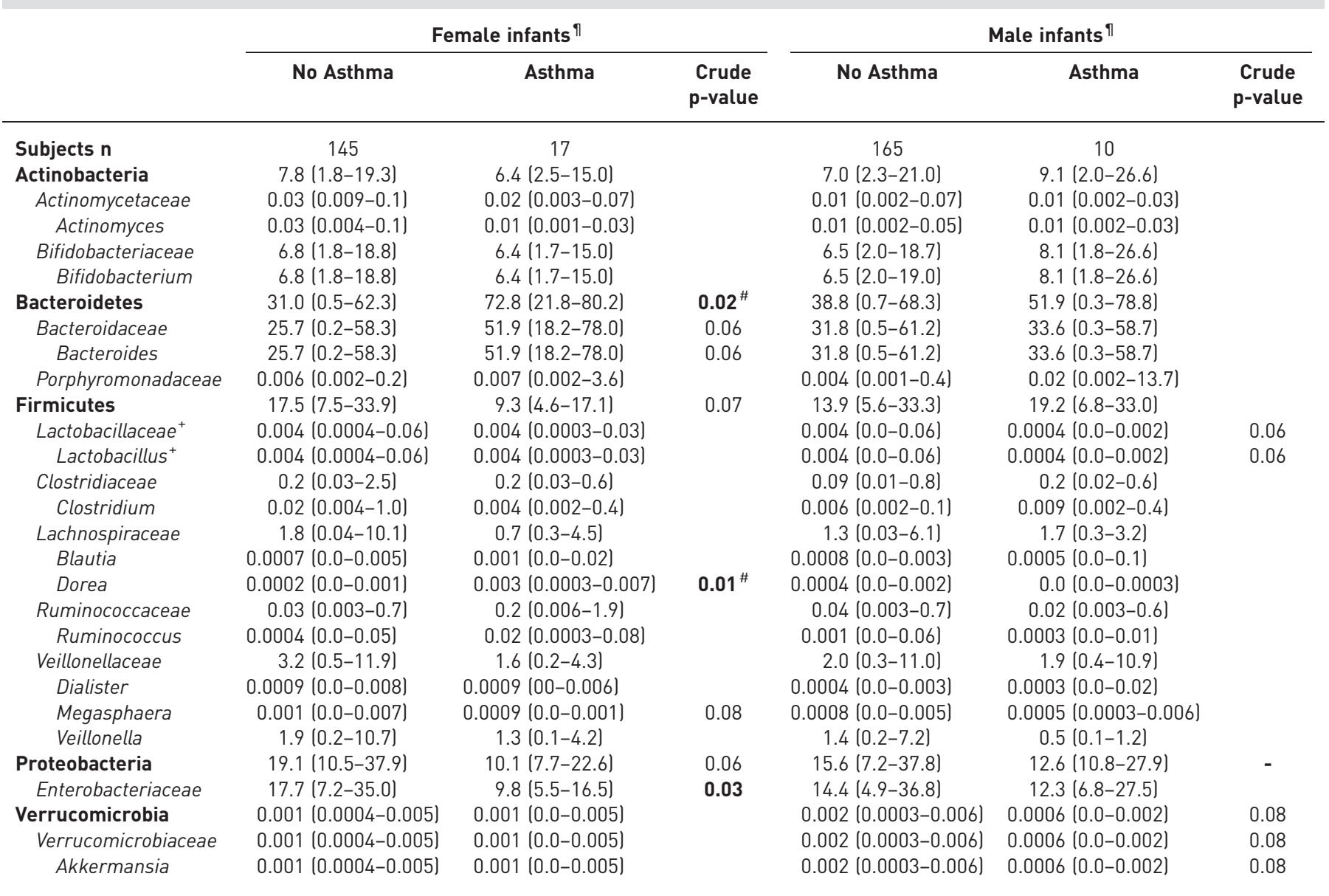

Data are presented as median (interquartile range), unless otherwise stated. Comparisons were performed using the Wilcoxon rank sum test. The false discovery rate (FDR) was used to adjust $p$-values for multiple testing and crude $p$-values <FDR p-values are indicated by "\#". The $p$-values $<0.1$ are reported and $p$-values $<0.05$ are indicated in bold. ${ }^{n}$ : restricted to Caucasian, vaginally delivered, breastfed infants not exposed to maternal antibiotics during birth; ${ }^{+}$: comparison of Lactobacillus was the primary hypothesis.

Adjustment for covariates in multi-variable regression showed the association between prenatal maternal asthma and gut lactobacilli in offspring to be quite robust (table 5). Independent of maternal atopy status and pre-pregnancy weight, birth mode, intrapartum antibiotic prophylaxis, breastfeeding status and sex, infants exposed to maternal asthma during pregnancy were one-third as likely to have Lactobacillus abundance in the highest tertile at 3-4 months of age. Statistical significance was confirmed in male (aOR, 0.26, 95\% CI 0.07-0.89), but not female infants (aOR, 0.48, 95\% CI 0.17-1.32). Regression models to predict the lowest tertile Lactobacillus abundance in infants as the outcome, showed no statistical associations with prenatal asthma status (data not shown). Only $2 \%$ of Caucasian infants received probiotic products; their exclusion from analyses did not change any of the findings.

Finally, using the LEfSe method to test differences at the species level (figure 1), an unclassified Lactobacillus species was found to be less abundant in male Caucasian infants born to mothers with atopic asthma (LDA score 2.93, $\mathrm{p}<0.05$ ). Unclassified Megasphera species (LDA score 3.34, $\mathrm{p}=0.003$ ) were comparatively less abundant and Bacteroides fragilis (LDA 3.29, $\mathrm{p}=0.02$ ) were more abundant in female Caucasian infants born to mothers with atopic asthma.

\section{Discussion}

In a birth cohort study of 1021 full-term Canadian infants, we detected alterations to gut microbial composition at 3-4 months following pregnancy complicated with asthma versus pregnancy without asthma. Infants of mothers with asthma harboured fewer lactobacilli in their gut microbiota, especially if 
TABLE 4 Median relative abundance of selected infant gut microbiota at 3-4 months, by maternal prenatal asthma, stratified by infant sex and maternal comorbid conditions during pregnancy

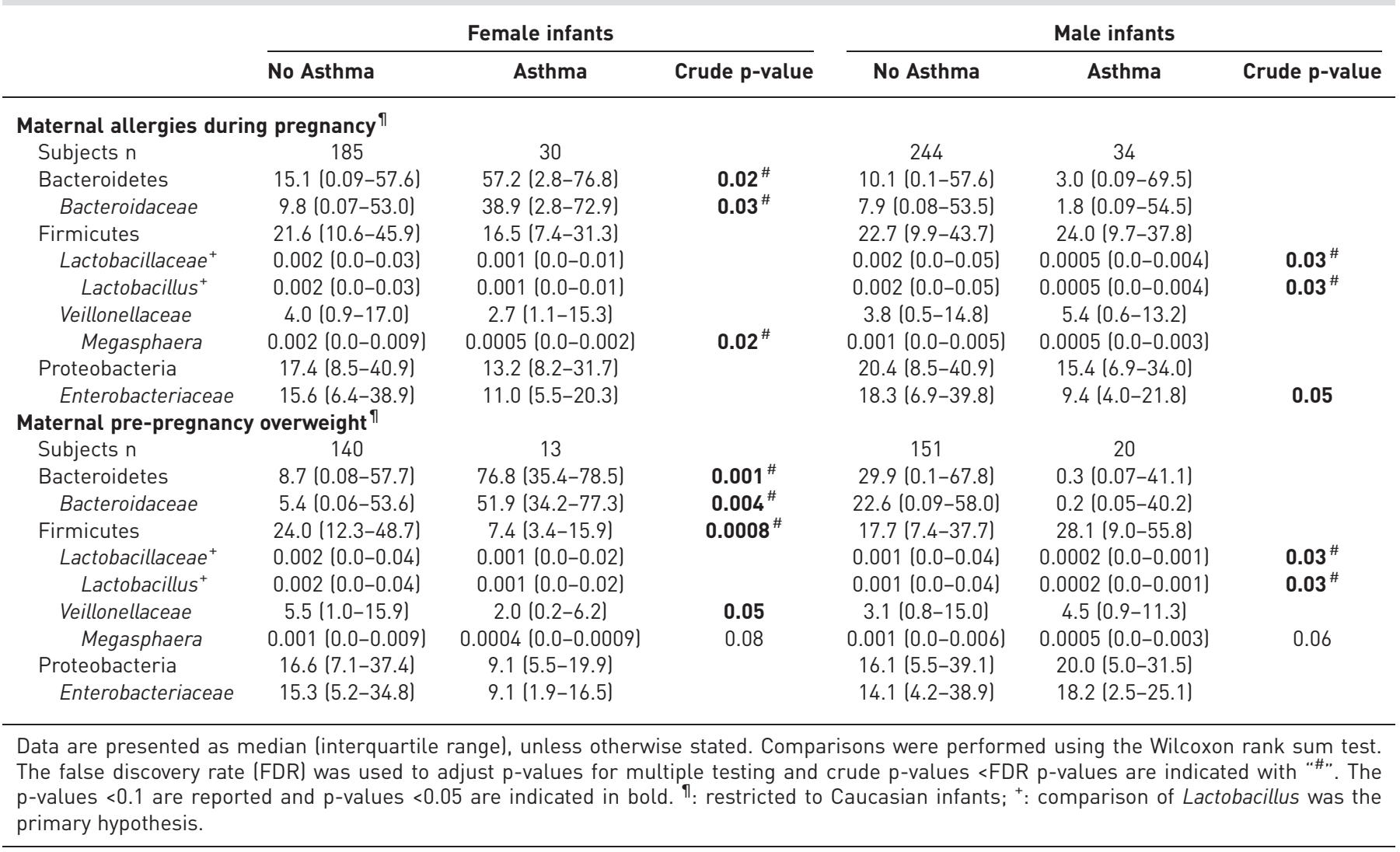

TABLE 5 Likelihood of highest tertile Lactobacillus abundance in the infant gut at 3-4 months of age according to maternal prenatal asthma, all children and sex-specific ${ }^{\#}$

\begin{tabular}{|c|c|c|c|c|c|}
\hline & Subjects $n$ & \multicolumn{2}{|c|}{ Crude models } & \multicolumn{2}{|c|}{ Adjusted models ${ }^{\eta}$} \\
\hline All infants & 710 & $0.35(0.16-0.74)$ & 0.006 & $0.36(0.16-0.78)$ & 0.01 \\
\hline Female infants & 324 & $0.45(0.17-1.19)$ & 0.11 & $0.48(0.17-1.32)$ & 0.16 \\
\hline Male infants & 386 & $0.26(0.08-0.86)$ & 0.02 & $0.26(0.07-0.89)$ & 0.03 \\
\hline
\end{tabular}

\#: analysis was restricted to Caucasian mothers; ": each model was adjusted for maternal pre-pregnancy weight, maternal food and environmental allergies during pregnancy, mode of delivery, maternal intrapartum antibiotic treatment, infant sex and breastfeeding status at 3-4 months. OR: odds ratio; aOR: adjusted odds ratio; $\mathrm{Cl}$ : confidence interval. The $\mathrm{p}$-values $<0.05$ are indicated in bold.

they were male and of Caucasian ethnicity $(\mathrm{p}=0.02)$. Moreover, the reduction in Lactobacillus species abundance was most apparent if Caucasian mothers with asthma had pre-pregnancy overweight or a history of food or environmental allergies. While no prior evidence exists for maternal prenatal asthma, these findings are compatible with the reduced Lactobacillales abundance observed in the meconium of newborns of mothers with eczema, and lower faecal colonisation with Lactobacillus species in infants of atopic parents $[14,28]$. In our study, male infants born to mothers with prenatal asthma were one-third (aOR 0.26, 95\% CI: 0.07-0.89) as likely to be colonised with high levels of lactobacilli, independent of maternal overweight or atopy status, birth mode or infant diet. These associations were not present in female offspring.

Male infants are at higher risk of developing wheeze during infancy than female infants, especially if they are Caucasian [27], although sex differences in gut microbiota composition have not been a focus of attention until recently [15]. Literature reporting a higher risk for overweight with antibiotic use in male, 

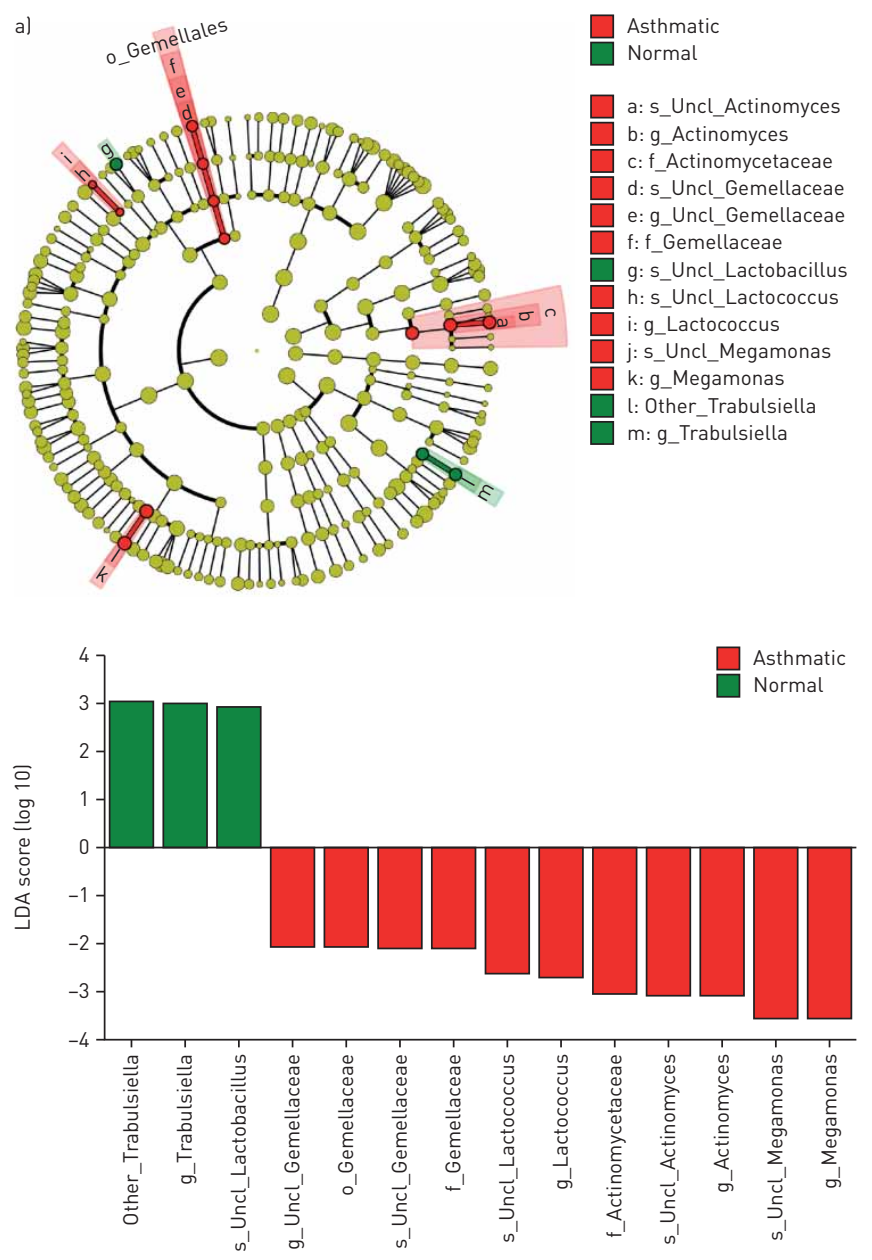
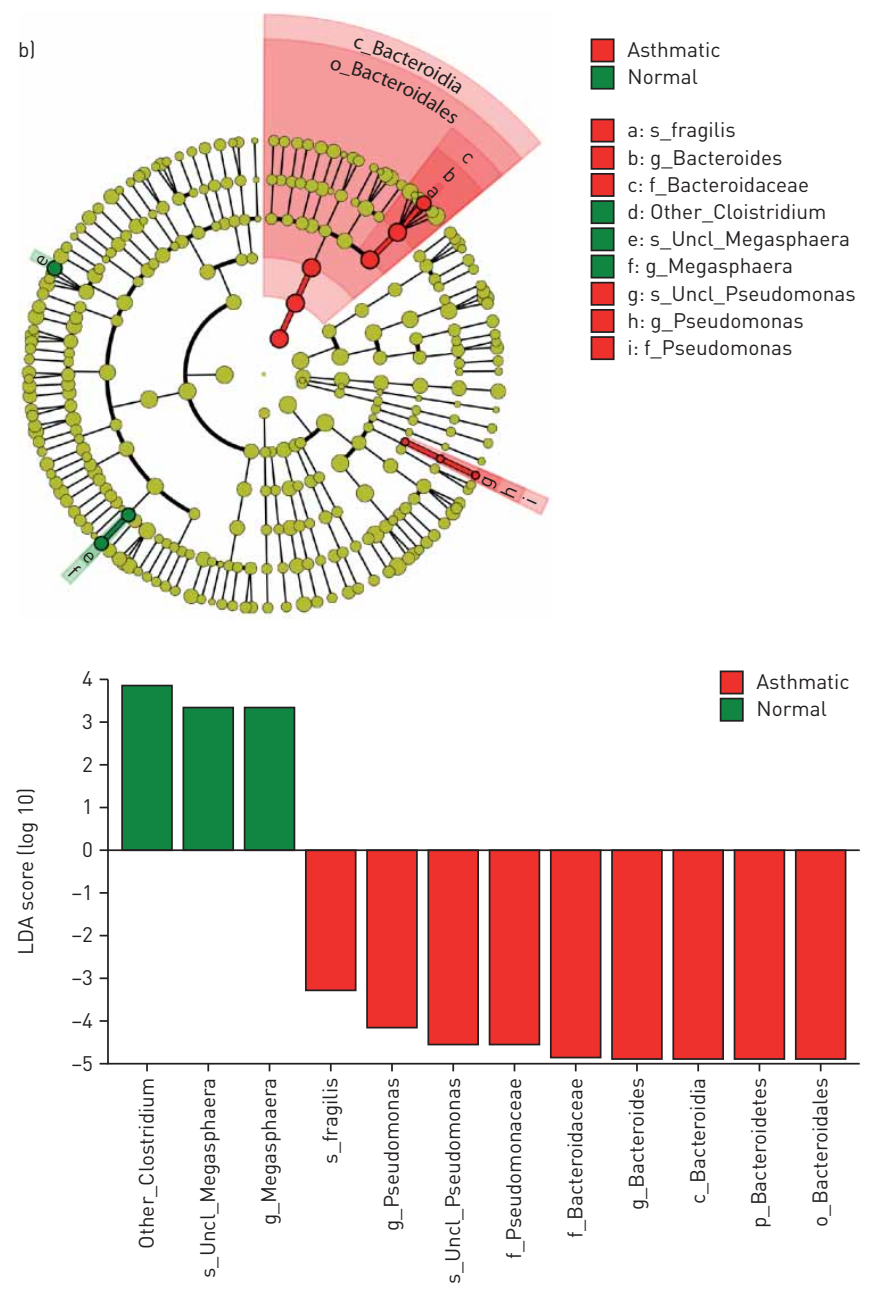

FIGURE 1 Cladogram and linear discriminant analysis (LDA) plots for prenatal maternal atopic asthma by a) male and b) female Caucasian infants from LDA effect size (LEfSe) methods.

but not female infants suggests differential sensitivity of male gut microbiota to environmental perturbations [17]. In their Australian Caucasian cohort, CuIfTon et al. [6] found that the response of the male placenta to maternal asthma exacerbations was minimal compared to that of the female placenta, with few changes to pro-inflammatory gene expression and 11ß-hydroxysteroid dehydrogenase activity in the placenta. This reduced response of the placenta heightened the risk for intrauterine growth restriction in male newborns. Noteworthy in our study is that no other significant changes to gut microbial composition were found for male infants born to asthmatic mothers, and Lactobacillus differences survived multiple correction. In alignment with evidence for prenatal asthma programming effects on infant growth, and the link between infant growth and gut microbial composition and atopic dermatitis [16, 30], our results hint at possible sexually dimorphic effects of prenatal asthma on infant gut microbiota.

As persistent residents of the human gastrointestinal tract in early infancy [31], lactate-producing lactobacilli are less abundant in the gut of infants who develop atopic disease by age 5 [32, 33]. Lactobacillus species are documented to elevate growth and immune factors in breast milk and anti-inflammatory cytokine levels in cord blood, when administered prenatally to the mother [34]; and postnatally, lactobacilli probiotics promote weight gain in infants [35]. A recent study by FARR et al. [36] observed birthweights of less than $2500 \mathrm{~g}$ to be more common following a mid-pregnancy vaginal microbiota dominated by non-lactobacilli versus lactobacilli microbes. Further, lactobacilli found in amniotic fluid are capable of modulating expression of toll-like receptor genes in the fetal gut [37]. In our study, the reduction in Lactobacillus abundance was most apparent among infants whose mothers had asthma and overweight or a history of allergies. Indeed, risk for atopic sensitisation at age 5 is reportedly elevated, with lower Lactobacillus colonisation by the age of 2 months in infants born to atopic parents [33]. Other high-risk cohort studies have found discordant faecal microbial biomarkers for atopic disease, such as 
lower abundance of Bacteroidetes or Ruminococcaceae, or higher abundance of the Clostridium cluster, but these observations were evident either at a much younger or older infant age; furthermore, sex-specific findings were not reported in these studies [38].

Maternal overweight during pregnancy has been linked to a higher risk of asthma and other atopic diseases in offspring $[39,40]$. In our study infants, maternal asthma comorbidity with this added risk for offspring also yielded a reduction in the abundance of faecal Megasphaera in female infants and an elevation in the abundance of Bacteroidaceae. The latter remained statistically significant after adjustment for the FDR; both species were identified as statistically more abundant by the LEfSe method. Both Megasphaera and Bacteroidaceae microbes produce propionate, but under opposing conditions. The Megasphaera species utilise lactate as a substrate to produce propionate [41], whereas Bacteroides species produce propionate when lactate levels are low [42]. Propionate is absorbed by the host for liver gluconeogenesis, possesses anti-inflammatory and satiety properties [43, 44], and protects against the development of allergic asthma in a murine model [45].

Lactobacillus bacteria are available to the newborn during vaginal delivery and breastfeeding, and are sensitive to antibiotic treatment $[10,46,47]$. Caesarean delivery and formula feeding have the potential to diminish maternal transfer of lactobacilli and other microbes to the infant $[13,48]$. Yet, the reduction in Lactobacillus abundance was also evident in male infants who were breastfed following vaginal delivery without maternal antibiotics, although statistical significance was lost after the reduction in sample size. Under the same circumstances, an enrichment of Bacteroidaceae and depletion of Enterobactericeae was detected in female microbiota following maternal prenatal asthma. Of interest, in the same cohort of infants, we found that a lower ratio of the faecal abundance of Enterobactericeae to Bacteroidaceae protected against food sensitisation [49]. Hence, our findings suggest a programming role for maternal asthma in shaping early gut colonisation, which cannot be attributed to caesarean delivery, antibiotic use or formula feeding, and which differs by sex and might confer less immediate risk for allergy in female infants [45].

There are several strengths to our research. Our findings were based on a large population-based cohort of full-term infants that included early-term infants, who are more likely to be delivered by asthmatic women and develop asthma [7, 50]. The large sample size also afforded stratification by covariates to identify associations between microbiota and maternal asthma, which were independent of birth-related events [28]. We adjusted p-values for multiple testing and verified sex and ethnicity interactions with a non-parametric test for interaction. High throughput gene sequencing enabled profiling of whole microbial communities in infant faecal samples, whereas most studies to date have targeted individual species. On the other hand, while the pregnancy questionnaire included criteria which had been validated as measures of prenatal asthma morbidity, maternal asthma status during pregnancy was not verified by a physician; neither did we test asthma severity, which might explain the modest impact of maternal asthma on birthweight [51].

Both prenatal and postnatal exposures are potential stimuli for health programming in the infant, through the modification of intestinal microbiota $[52,53]$. Initial colonisation of the neonatal gut plays a central role in the development of the immune system [54]. Our results provide the first evidence of a programming effect of prenatal maternal asthma on gut microbial composition that is independent of birth and postnatal events. In addition to smaller lung size at birth [20] or other risk factors for wheeze [27], reductions in gut lactobacilli point to another pathway for persistent asthma in male Caucasian infants. Having fewer intestinal lactobacilli also has additional implications for male infants, such as reduced resistance to pathogens [55]; whereas gut microbial changes, such as an increased abundance of Bacteroidaceae, might have a protective effect in female infants.

\section{Acknowledgements}

We are grateful to all the families who took part in this study, and the whole CHILD team, which includes interviewers, computer and laboratory technicians, clerical workers, research scientists, volunteers, managers, receptionists and nurses. We thank Sarah Bridgman for her assistance in formatting the final version of the manuscript and Konstantin Shestopaloff for completing the ART tests. Anita Kozyrskyj will serve as the guarantor for the contents of this paper.

CHILD Study Investigators: SS Anand, McMaster University; MB Azad, University of Manitoba; AB Becker, University of Manitoba; AD Befus, University of Alberta; M Brauer, University of British Columbia; JR Brook, University of Toronto; E Chen, Northwestern University, Chicago; MM Cyr, McMaster University; D Daley, University of British Columbia; SD Dell, The Hospital for Sick Children and University of Toronto; JA Denburg, McMaster University; QL Duan, Queen's University; T Eiwegger, The Hospital for Sick Children and University of Toronto; H Grasemann, The Hospital for Sick Children and University of Toronto; K HayGlass, University of Manitoba; RG Hegele, The Hospital for Sick Children and University of Toronto; DL Holness, University of Toronto; P Hystad, Oregon State University; M Kobor, University of British Columbia; TR Kollmann, University of British Columbia; AL Kozyrskyj, University of Alberta; C Laprise, Université du Québec à Chicoutimi; WYW Lou, University of Toronto; J Macri, McMaster University; PJ Mandhane, University of Alberta; G Miller, Northwestern University, Chicago; TJ Moraes, The Hospital for Sick Children and University of Toronto; P Paré, University of British Columbia; C Ramsey, University of 
Manitoba; F Ratjen, The Hospital for Sick Children and University of Toronto; A Sandford, University of British Columbia; J Scott, University of Toronto; JA Scott, University of Toronto; MR Sears (Founding Director), McMaster University; F Silverman, University of Toronto; E Simons, University of Manitoba; P Subbarao (Director), The Hospital for Sick Children and University of Toronto; T Takaro, Simon Fraser University; SJ Tebbutt, University of British Columbia; T To, The Hospital for Sick Children and University of Toronto; SE Turvey (Co-director), University of British Columbia.

Author contributions: P.T. Koleva performed statistical analysis, prepared figures and tables, and wrote the first draft of the manuscript. A.L. Kozyrskyj conceived the study and obtained funding, designed the analyses and wrote the final version of the manuscript. H.M. Tun conducted the LEfSE analyses. Under the supervision of J.A. Scott, T. Konya conducted DNA extraction and sample preparation for sequencing in the laboratory of D. Guttman. Microbiota bioinformatics was conducted by J.A. Scott, D. Guttman, A.B. Becker, P.J. Mandhane, P. Subbarao, S.E. Turvey; and M. R. Sears helped obtain funding, advised on the study design, and coordinated data collection. All authors provided critical comments on the manuscript content and approved the final version of the manuscript.

\section{References}

1 Sawicki E, Stewart K, Wong S, et al. Medication use for chronic health conditions by pregnant women attending an Australian maternity hospital. Aust N Z J Obstet Gynaecol 2011; 51: 333-338.

2 Pali-Scholl I, Motala C, Jensen-Jarolim E. Asthma and allergic diseases in pregnancy: a review. World Allergy Organ J 2009; 2: 26-36.

3 Ali Z, Ulrik CS. Incidence and risk factors for exacerbations of asthma during pregnancy. J Asthma Allergy 2013; 6: 53-60.

4 Murphy VE, Schatz M. Asthma in pregnancy: a hit for two. Eur Respir Rev 2014; 23: 64-68.

5 Clifton VL, Engel P, Smith R, et al. Maternal and neonatal outcomes of pregnancies complicated by asthma in an Australian population. Aust NZ J Obstet Gynaecol 2009; 49: 619-626.

6 Clifton VL, Davies M, Moore V, et al. Developmental perturbation induced by maternal asthma during pregnancy: the short- and long-term impacts on offspring. J Pregnancy 2012; 2012: 741613.

7 Rejno G, Lundholm C, Gong $\mathrm{T}$, et al. Asthma during pregnancy in a population-based study-pregnancy complications and adverse perinatal outcomes. PLoS One 2014; 9: e104755.

8 Martel MJ, Rey E, Beauchesne MF, et al. Control and severity of asthma during pregnancy are associated with asthma incidence in offspring: two-stage case-control study. Eur Respir J 2009; 34: 579-587.

9 Stensballe LG, Simonsen J, Jensen SM, et al. Use of antibiotics during pregnancy increases the risk of asthma in early childhood. J Pediatr 2013; 162: 832-838.

10 Koren O, Goodrich JK, Cullender TC, et al. Host remodeling of the gut microbiome and metabolic changes during pregnancy. Cell 2012; 150: 470-480.

11 Konstantinov SR, van der Woude CJ, Peppelenbosch MP. Do pregnancy-related changes in the microbiome stimulate innate immunity? Trends Mol Med 2013; 19: 454-459.

12 Doyle RM, Alber DG, Jones HE, et al. Term and preterm labour are associated with distinct microbial community structures in placental membranes which are independent of mode of delivery. Placenta 2014; 35: 1099-1101.

13 Hall MA, Cole CB, Smith SL, et al. Factors influencing the presence of faecal lactobacilli in early infancy. Arch Dis Child 1990; 65: 185-188.

14 Gosalbes MJ, Llop S, Valles Y, et al. Meconium microbiota types dominated by lactic acid or enteric bacteria are differentially associated with maternal eczema and respiratory problems in infants. Clin Exp Allergy 2013; 43: 198-211.

15 Martin R, Makino H, Cetinyurek YA, et al. Early-life events, including mode of delivery and type of feeding, siblings and gender, shape the developing gut microbiota. PLoS One 2016; 11: e0158498.

16 O'Donovan SM, O'B Hourihane J, Murray DM, et al. Neonatal adiposity increases the risk of atopic dermatitis during the first year of life. J Allergy Clin Immunol 2016; 137: 108-117.

17 Kozyrskyj AL, Kalu R, Koleva PT, et al. Fetal programming of overweight through the microbiome: boys are disproportionately affected. J Dev Orig Health Dis 2016; 7: 25-34.

18 Million M, Angelakis E, Paul M, et al. Comparative meta-analysis of the effect of Lactobacillus species on weight gain in humans and animals. Microb Pathog 2012; 53: 100-108.

19 Moraes TJ, Lefebvre DL, Chooniedass R, et al. The Canadian healthy infant longitudinal development birth cohort study: biological samples and biobanking. Paediatr Perinat Epidemiol 2015; 29: 84-92.

20 Subbarao P, Anand SS, Becker AB, et al. The Canadian Healthy Infant Longitudinal Development (CHILD) study: examining developmental origins of allergy and asthma. Thorax 2015; 70: 998-1000.

21 Takaro TK, Scott JA, Allen RW, et al. The Canadian Healthy Infant Longitudinal Development (CHILD) birth cohort study: assessment of environmental exposures. J Expo Sci Environ Epidemiol 2015; 25: 580-592.

22 Schatz M, Dombrowski MP, Wise R, et al. Asthma morbidity during pregnancy can be predicted by severity classification. J Allergy Clin Immunol 2003; 112: 283-288.

23 Belanger K, Hellenbrand ME, Holford TR, et al. Effect of pregnancy on maternal asthma symptoms and medication use. Obstet Gynecol 2010; 115: 559-567.

24 Bokulich NA, Subramanian S, Faith JJ, et al. Quality-filtering vastly improves diversity estimates from Illumina amplicon sequencing. Nat Methods 2013; 10: 57-59.

25 Banjamini Y, Hochberg Y. Controlling the false discovery rate: a practical and powerful approach to multiple testing. J Royal Stat Soc 1995; 51: 289-300.

26 Yadav D, Ghosh TS, Mande SS. Global investigation of composition and interaction networks in gut microbiomes of individuals belonging to diverse geographies and age-groups. Gut Pathog 2016; 8: 17.

27 Garcia-Marcos L, Mallol J, Sole D, et al. International study of wheezing in infants: risk factors in affluent and non-affluent countries during the first year of life. Pediatr Allergy Immunol 2010; 21: 878-888.

28 Azad MB, Konya T, Persaud RR, et al. Impact of maternal intrapartum antibiotics, method of birth and breastfeeding on gut microbiota during the first year of life: a prospective cohort study. BJOG 2016; 123: 983-993.

29 Leys C, Schumann S. A nonparametric method to analyze interactions: the adjusted rank transform test. J Exp Soc Psychol 2010; 46: 684-688. 

microbiota that affect infant growth. PLoS Comput Biol 2013; 9: e1003042. Infect 2005; 7: 1256-1262.

32 Penders J, Thijs C, Mommers M, et al. Intestinal lactobacilli and the DC-SIGN gene for their recognition by dendritic cells play a role in the aetiology of allergic manifestations. Microbiology 2010; 156: 3298-3305.

33 Johansson MA, Sjogren YM, Persson JO, et al. Early colonization with a group of Lactobacilli decreases the risk for allergy at five years of age despite allergic heredity. PLoS One 2011; 6: e23031.

34 Prescott SL, Wickens K, Westcott L, et al. Supplementation with Lactobacillus rhamnosus or Bifidobacterium lactis probiotics in pregnancy increases cord blood interferon-gamma and breast milk transforming growth factor-beta and immunoglobin A detection. Clin Exp Allergy 2008; 38: 1606-1614.

35 Robinson EL, Thompson WL. Effect on weight gain of the addition of Lactobacillus acidophilus to the formula of newborn infants. J Pediatr 1952; 41: 395-398.

36 Farr A, Kiss H, Hagmann M, et al. Role of Lactobacillus species in the intermediate vaginal flora in early pregnancy: a retrospective cohort study. PLoS One 2015; 10: e0144181.

37 Rautava S, Collado MC, Salminen S, et al. Probiotics modulate host-microbe interaction in the placenta and fetal gut: a randomized, double-blind, placebo-controlled trial. Neonatology 2012; 102: 178-184.

38 Bridgman SL, Kozyrskyj AL, Scott JA, et al. Gut microbiota and allergic disease in children. Ann Allergy Asthma Immunol 2016; 116: 99-105.

39 Forno E, Young OM, Kumar R, et al. Maternal obesity in pregnancy, gestational weight gain, and risk of childhood asthma. Pediatrics 2014; 134: e535-e546.

40 Ekstrom S, Magnusson J, Kull I, et al. Maternal body mass index in early pregnancy and offspring asthma, rhinitis and eczema up to 16 years of age. Clin Exp Allergy 2015; 45: 283-291.

41 Duncan SH, Louis P, Flint HJ. Lactate-utilizing bacteria, isolated from human feces, that produce butyrate as a major fermentation product. Appl Environ Microbiol 2004; 70: 5810-5817.

42 Macfarlane S, Macfarlane GT. Regulation of short-chain fatty acid production. Proc Nutr Soc 2003; 62: 67-72.

43 Walker AW, Duncan SH, McWilliam Leitch EC, et al. pH and peptide supply can radically alter bacterial populations and short-chain fatty acid ratios within microbial communities from the human colon. Appl Environ Microbiol 2005; 71: 3692-3700.

44 Shetty SA, Marathe NP, Lanjekar V, et al. Comparative genome analysis of Megasphaera sp. reveals niche specialization and its potential role in the human gut. PLoS One 2013; 8: e79353.

45 Trompette A, Gollwitzer ES, Yadava K, et al. Gut microbiota metabolism of dietary fiber influences allergic airway disease and hematopoiesis. Nat Med 2014; 20: 159-166.

46 Soto A, Martin V, Jimenez E, et al. Lactobacilli and Bifidobacteria in human breast milk: influence of antibiotherapy and other host and clinical factors. J Pediatr Gastroenterol Nutr 2014; 59: 78-88.

47 Jost T, Lacroix C, Braegger CP, et al. Vertical mother-neonate transfer of maternal gut bacteria via breastfeeding. Environ Microbiol 2014; 16: 2891-2904.

48 Fallani M, Young D, Scott J, et al. Intestinal microbiota of 6-week-old infants across Europe: geographic influence beyond delivery mode, breast-feeding, and antibiotics. J Pediatr Gastroenterol Nutr 2010; 51: 77-84.

49 Azad MB, Konya T, Guttman DS, et al. Infant gut microbiota and food sensitization: associations in the first year of life. Clin Exp Allergy 2015; 45: 632-643.

50 Harju M, Keski-Nisula L, Georgiadis L, et al. The burden of childhood asthma and late preterm and early term births. J Pediatr 2014; 164: 295-299.

51 Murphy VE, Namazy JA, Powell H, et al. A meta-analysis of adverse perinatal outcomes in women with asthma. BJOG 2011; 118: 1314-1323.

52 West CE, Renz H, Jenmalm MC, et al. The gut microbiota and inflammatory noncommunicable diseases: associations and potentials for gut microbiota therapies. J Allergy Clin Immunol 2015; 135: 3-13.

53 Koleva PT, Kim JS, Scott JA, et al. Microbial programming of health and disease starts during fetal life. Birth Defects Res C Embryo Today 2015; 105: 265-277.

54 Maynard CL, Elson CO, Hatton RD, et al. Reciprocal interactions of the intestinal microbiota and immune system. Nature 2012; 489: 231-241.

55 Munoz-Quezada S, Bermudez-Brito M, Chenoll E, et al. Competitive inhibition of three novel bacteria isolated from faeces of breast milk-fed infants against selected enteropathogens. Br J Nutr 2013; 109: Suppl. 2, S63-S69. 